\title{
E
}

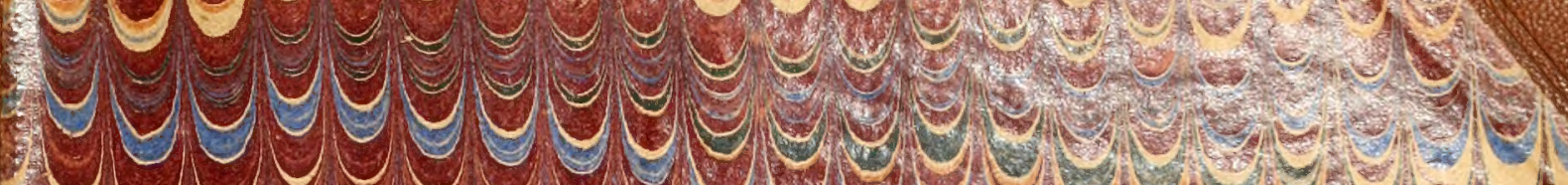

C e c a s ren.

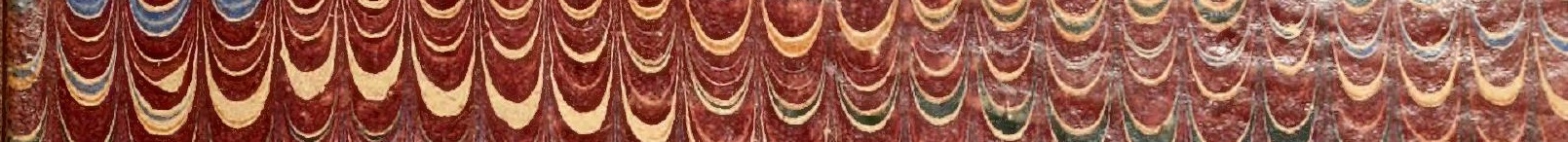
280 _ a a 30 S _ a Ca S _e

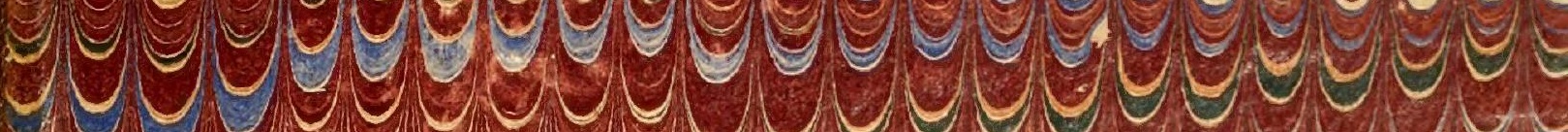
Y

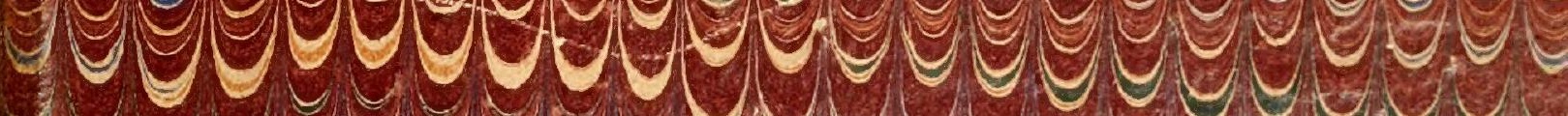
C

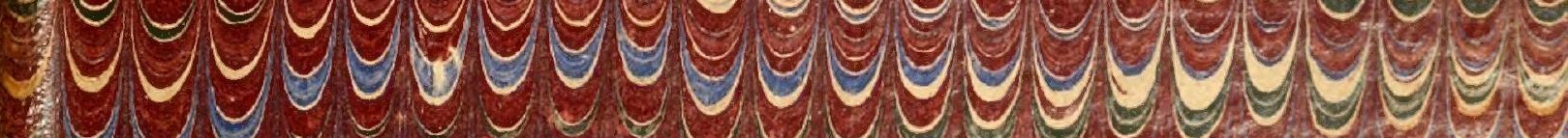

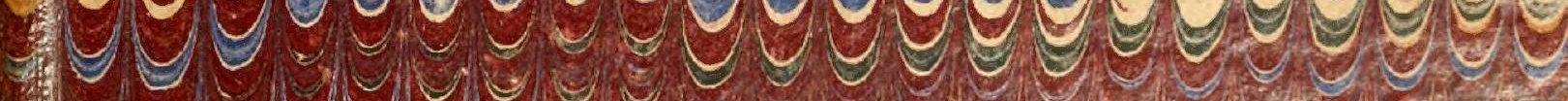

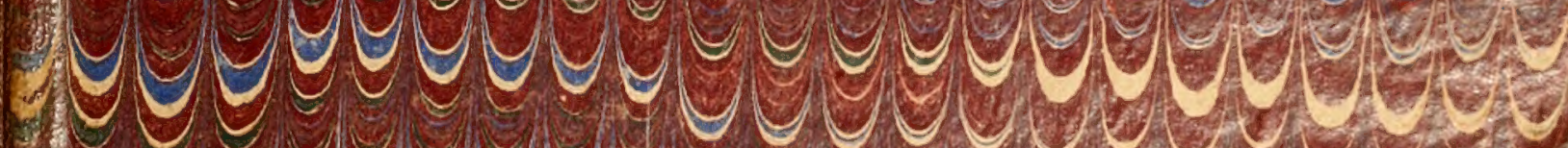

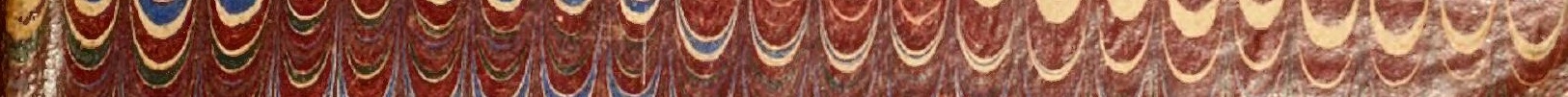

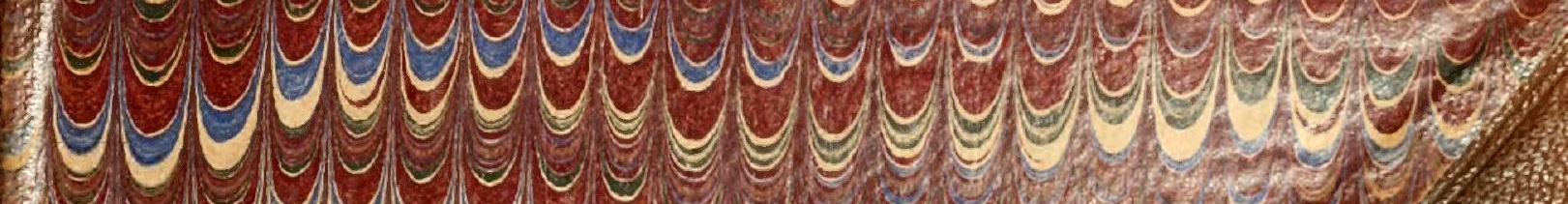




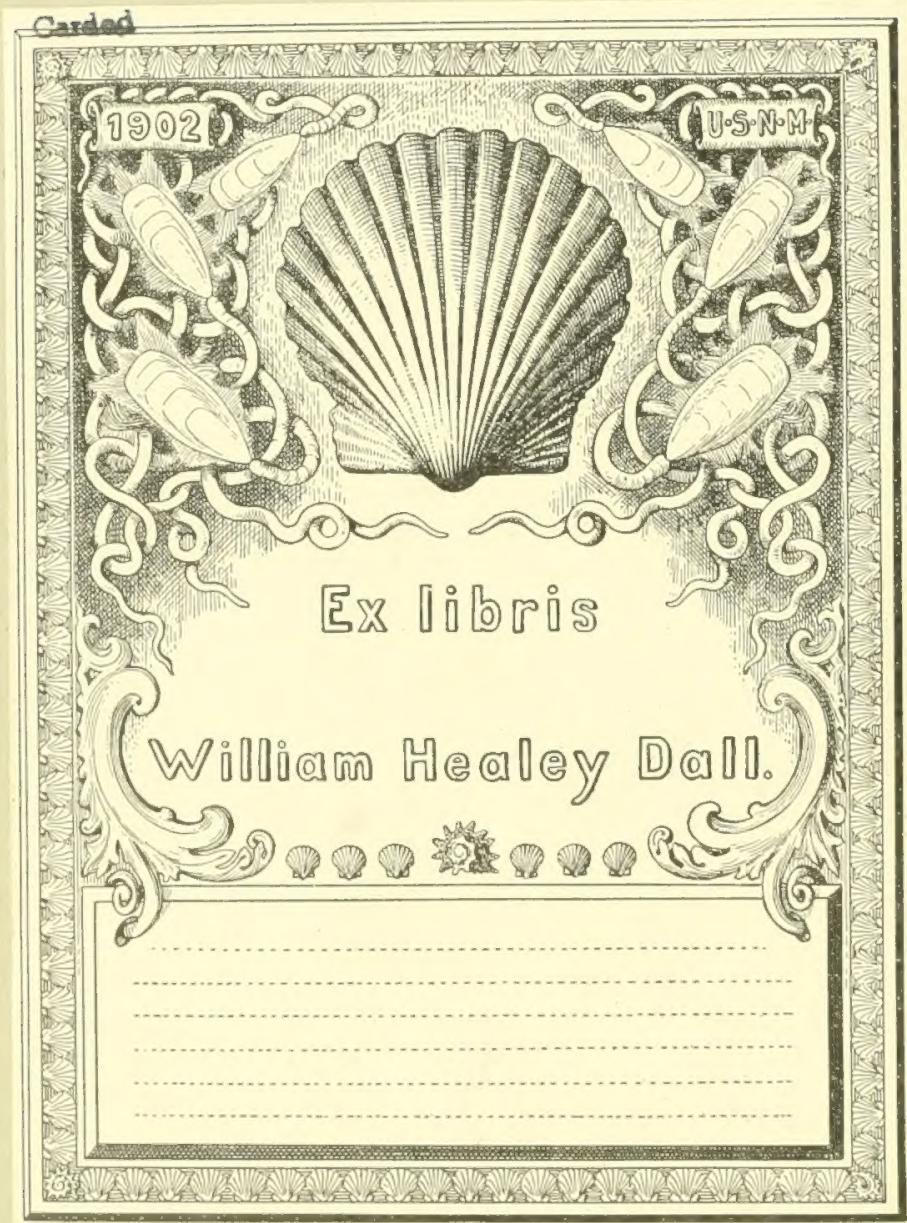







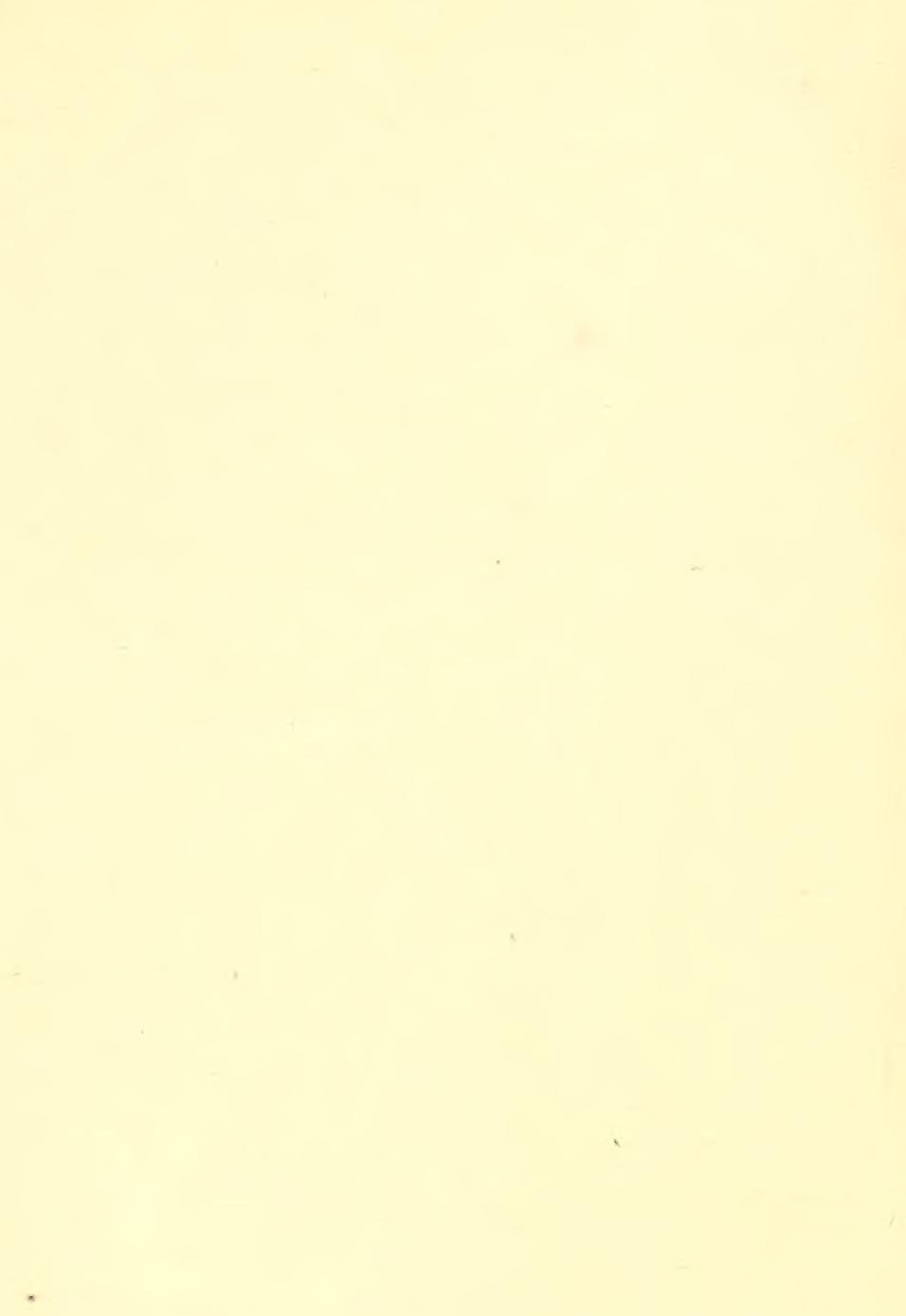






\title{
Cenni intorno alle Verticordie fossili tel Plioceno italiano
}

NoT $\mathbf{A}$

\author{
del Socio Corrispondente nazionale G. Seguenza
}

(Adunanza del di 6 maggio $\mathbf{1 8 7 6}$

Estratto diri Rendiconto della Reate Accademia delle Scienze fisiche e matematiche

Fascicolo $6^{0}$ - Giugno 1876

L'Hippagus acuticostatus, Philippi, che trovasi non troppo raro nel plioceno messinese, fu descritto incompletamente dal suo scuopritore, il quale conobbe soltanto la sinistra valva, perlochè al 1860 io era indotto, dai belli esemplari posseduti, a dare una completa descrizione di questa elegante specie nel Journal de Conchyliologie di Parigi ${ }^{1}$ ), e dallo studio di tutti i suoi caratteri a rapportarla al genere Verticordia, S. Wood, al quale vi riferiva benanco un' altra specie, che denominai $V$. granulata.

Da quell'epoca sinoggi il genere Verticordia si è andato arricchendo grado grado di specie nuove e sempre più variate, scoperte negli strati dell'Eoceno, del Mioceno, del Plioceno e nei mari attuali.

Nell' Eoceno conoscesi la $V$. parisiensis, e forse anco vi si deve riferire l' Hippagus Leanus, ambedue descritte dal Deshayes.

Nel mioceno torinese si è raccolta la Chama arietina del Brocchi, per la quale il Prof. Meneghini avea fondato il genere Pecchiolia, ma essa è evidentemente congenere alla $V$. acuticostata, la quale per forme intermedie varie si connette naturalmente a specie poco convesse e cogli apici non spirali, perlocchè la grande gibbosità delle valve e l'avvolgimento spirale degli apici non valgono a separare genericamente la Chama arietina, la quale madreperlacea nell' interno offre un grosso dente sulla destra valva, ed una profonda scanalatura nell'interno del margine dorsale di ambedue, per l' inserzione del ligamento, caratteri che distinguono tutte le Verticordie conosciute.

Nel mioceno torinese trovasi anco una bivalve madreperlacea, finamente costata, cogli apici avvolti e con una grande area depressa cordiforme sulla regione boccale, che venne denominata Pecchiolia Gastaldi dal Michelotti. Questa specie ancora devesi a mio credere riferire al genere Verticordia.

Nel plioceno italiano sinora pare che siansi raccolte la Chama arietina, Brocch i, l'Hippagus acuticostalus, Phil, , la Verticordia granulata, Seg., e la Pecchiolia arenosa, Rayneval, V anden Hecke e Ponzi, le quali specie tutte senza dubbio spettano al genere Verticordia.

La $V$. cardiiformis, S. Wood, del Grag corallino di Sutton, è quella per la quale fu fondato tal genere, e parmi che oggi sia ben assicurato che tale specie sia l'identica dell' Hippagus acuticostatus del Philippi.

1) Du genre Verticordia (Tom. 8, pag 291 ). 
Le specie viventi poi si sono considerevolmente aumentate. La Trigonulina ornata del D' O rbigny è una Verticordia che vive alla Giammaica, e che più recentemente fu descritta da Adams e Reève col nome d'Hippagus novemostatus vivente nei mari della China.

La $V$. cardiiformis, S. Wood, fu pescata nei mari della China e descritta da P. Fischer col nome di $V$. Deshayesiana, da A. Adams tra le conchiglie del Giappone come V. Japonica.

La V.granulata, Seguenza, fu identificata a quella pescata nei mari del Giappone e deseritta col nome di V. multicostata, A. Adams, ma tale identificazione è almeno dubbia.

Non ha guari nei mari d' Europa il genere Verticordia vi era affatto sconosciuto, ma grazie alle moderne investigazioni, e sopratutto alle pesche nelle grandi profondita dell' Atlantico e del Mediterraneo si conoscono ormai la Verticordia acuticostata, Philippi, nell' Atlantico e nel Nordatlantico, la $V$. abyssicola, Sars, nei mari del Nord-Europa. La $V$. insculpta, Jeffreys, pescata presso le coste d'Algeria (Jeffreys) ed a Palermo (Monterosato), dove furono insieme trovati taluni frammenti della Pecchiolia arenosa, Rayn? (Monterosato). Il signor March. di Monterosato comunicavami gentilmente per mezzo di lettera di aver pescata recentemente nei mari di Palermo una nuova specie, che io avea già raccolta nel plioceno e denominata $V$. trapezoidea; tale specie pescata nel Mediterraneo e in molti luoghi dell'Atlantico fu creduta la $V$. granulata, che forse ancora non si conosce vivente.

Avendo raccolto nel plioceno messinese e di Calabria alcune nuove specie di Verticordia mi sono proposto fin da molto tempo di dare una monografia di tutte le specie plioceniche, descrivendole ed illustrandole accuratamente; ma siccome per varie ragioni trascorrerà ancora del tempo fino a che un tale mio proposito sia mandato ad effetto, credo utile pubblicare un cenno intorno alle distinzioni specifiche, ed ai luoghi dove ciascuna specie è stata raccolta.

Sin' ora sono quattro le specie conosciute nel plioceno italiano come precedentemente ho detto, due delle quali esclusive delle provincie meridionali e le altre due ve l'ho raccolte ancora recentemente; ma inoltre ho scoperto in quegli strati del plioceno antico, di unita alle precedenti, altre cinque specie variissime che sono del più alto interesse per la conoscenza delle modificazioni specifiche che subisce questo genere, il quale a ragione può dirsi polimorfo.

Eccomi dunque all'enumerazione delle Verticordie plioceniche, che sembrami naturalissimo dovere aggruppare in tre distinti sottogeneri.

Genere-VERTICORDIA, S. Wood.

Specie del Pliocenoitaliano

PRIMO SOTTOGENERE.

Pecchiolia, M eneghini, 1852. - Conchiglia costata, cogli apici assai grandi, prominenti ed avvolti a spirale, dente grosso prominente trigono, rivolto verso la regione anale e disposto parallelamente al margine dorsale; solco interno al margine dorso-anale ben distinto, lunula non distinta.

Di questa sezione si conoscono soltanto le due specie seguenti:

Prima Specie - VERTICORDIA ARGENTEA

Sinonimia

1797 - Chama argentea, Mariti - Odeporico. Vol. I, p. 524, gen. 511, 11.015.

1814 - Chama arietina, Brocchi - Conch. foss. subappen. ec. II, p. 668, tav. XVI, fig. 13, $a, b$. 1815-Isocardia arietina, Lamarck. - Animaux sans vert. Vol. VI, pag. 146.

1831 - Isocardia arietina, Bronn. - Ital. tert. geb., pag. 106, n. ${ }^{\circ} 610$. 
1847 - Hippagus arietinus (Bellardi) Sismonda.-Synopsis meth. an. ped. foss.. pag. 18. 1847 - Isocardia arietina, Michelotti - Description des foss. mioc. ec., pag. 99, tav. 4, fig. 22. 1851 - Pecchiolia argentea, Savi e Me neghini - Cons. sulla geologia stratigraf. della Toscana. pag. 45 \&.

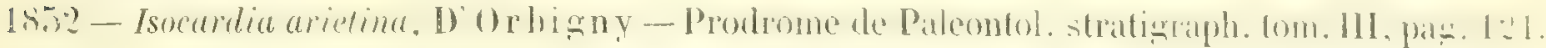

1852 - Pecchiolia urgentea, V. Pecchioli-Notice sur un nouveau genre de bivalve foss. des terr. sub. (Révue et Magasin de Zoologie; 2e Serie, Tome IV, tav. 13, fig. 1-4).

1855 - Isocardia arietina, l'ictet - Traité de paleontologic. Tom. III, p. 482.

1862 - Pecchiolia argentea, Hoernes - Die foss. moll. des tert. Beck. von IVien. II. Bd., 3-i Lleft, tav. 20, fig. 4, $a, d$.

1S71 - Pecchiolia argentea, F. L. Appelius - Cat. delle Conch. foss. del Livornese, pag. 102. 1873 - Pecchiolia argentea, G. Cocconi-Enum. sist. dei moll. mioc. e plioc. delle prov. di Parma e di Piacenza, pag. 308.

1871-Pecchiolia argentea, L. Foresti - Catalogo dei molluschi foss. plioc. delle coll. bolognesi, p. II, pag. 27.

1875-? Pechiolia Mariti, G. Ponzi - Cronaca subappennina, pag. 9.

Questa distinta e ben nota specie, che è stata scoperta sin dallo scorso secolo, che venne ti-

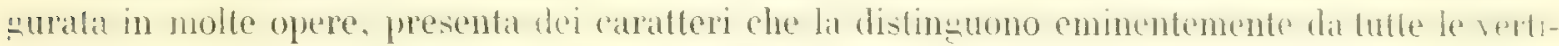
cordie conosciute. Le valve sono massimanente convesse a i lopo apici prominentisimi suno l:

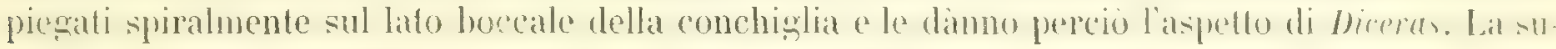
prerlicie esterma é percorsa da costole radianti, obliquanente incurvate, olluse, piecole nume-

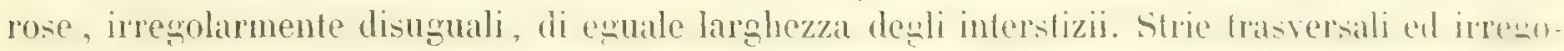
lari di acerescimento na poco distinte segnano la superticie, lat quale e inoltre dapuertulto zrannsil, coperta da granuli piecoli e rotondati. Il dente sulla destral valva conico-compresso, obligulu-

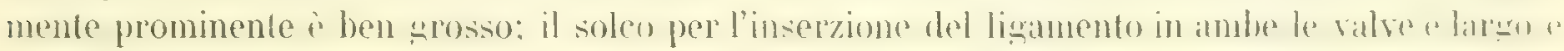
profondo.

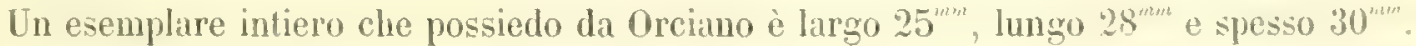

Giacimento. - Fu riconosciuta dal Michelotti, dal Bellardi, dal Sismonda nel Hioceno medio della collina di 'lorino (C.S. $)^{1}$ ).

Fu raccolta anco nel mioceno superiore del Bacino di Viema (I oernes), come del Modenese (Doderlein) e del Tortonese (Michelotti) (C. S.).

lo stesso, ho trovato un modello ben riconoscibile ai Bianchi (T'err." di Messina) in una moLassa del mioceno superiore (C. S.).

Yella zona superiore dell'antico plioceno questa specie giace quasi dipertutto in Italia.

Fu raccolta nel Piacentino (Brocchi, Michelotti, Coceoni), ad Orciano (Pecchioli) (C.S.), nel Bolognese (Foresti), ad Albaro presso Parma, ad Lmiano presso Genova (Marc. Pareto), presso Livorno (Caterini, Appelius), al Honte Valicano (Ponzi).

Per quanto io conosca non è stata segnalata nell'Italia meridionale, ed io la raccoglieva re-

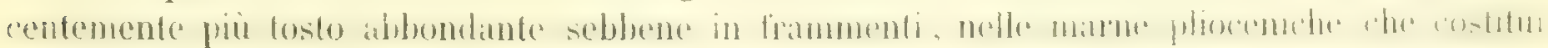
scono le colline che cingono. Bianconuovo nella Provincia di kegrio, siccome presso (hioiosa jonica, Riace, e Mlonosterace nella medesima provincia (C. S.).

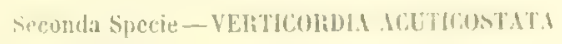

Sinonimia

1840 - Cryptodon? verticordia, S. Wood-Ciat. Brit. mus.

1814 -Verticordia cardiiformis, S. Wood (II. S.). 
1844 - Verticordia cardifformis, Sowerby. - Min. conch. Tav. 639.

1844-Mippagus cardiformis, Sowerby - Min. conch. ('Testo).

1844 - Hippagus acuticostatus, Philippi-Enumeratio moll. Siciliae. Vol. II , pag. 11 , Iav. XIV, fig. 18.

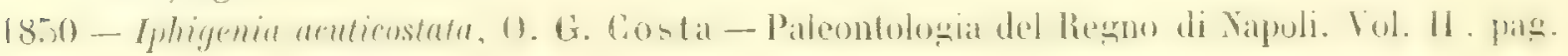
160, t. 13 , fig. 9 .

1851 - Hippagus verticordius, S. Wood-Mollusca from the crag.; pag. 150, tav. 12, fig. $18, a, b$.

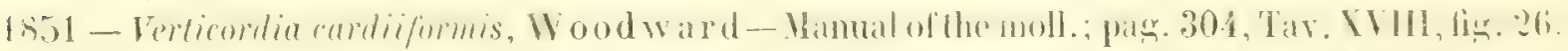

1858 - Verticordia acuticostata, G. Seguenza - Del genere Verticordia, Wood.- Breve monografia delle specie foss. di Sicilia-Eco peloritano, anno V, fasc. 5.

1860 - Verticordia aculicostata, Seguenza-Du genre Verticordia (Journal de Conch., tom. 8, pag. 291, Tav. 10, lig. 1, $a, b, c, d, e)$.

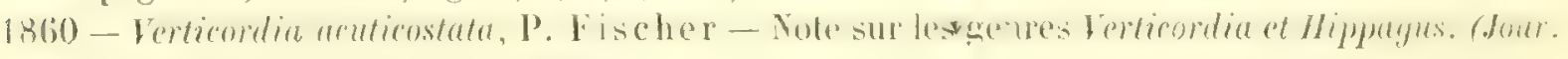
de Conchiliol. Vol. 8, pag. 299.

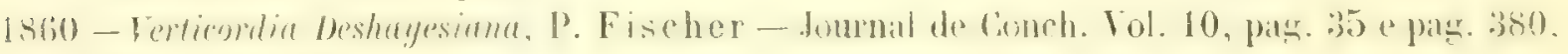
Tav. 5, fig. 10, 11.

1860 -Verticordin cardiiformis, P. Fischer - Journal de Conch. Vol. 8, pag. 299; Vol. 10. pag. 380.

1862 -Verticordia acuticostata, P. F is cher-Journal. de Conch. Vol. 10, p. 381.

1862 -Verticorlia Japonica, A. Adams. - On some new. sper of acephalous Moll. from the sea of Japon ( $\mathrm{nnn}$. and magan. of nat. history. p. 224.

1862 - Verticordia Japonica, P. Fische r. - Journal de Conch. Tom. 10, p. 378 e 380.

1862 - Verticordia aculienstala, Sesuenza - Notizie stucemte intorno la cost. geol. del distrutto di Messina, p. 18.

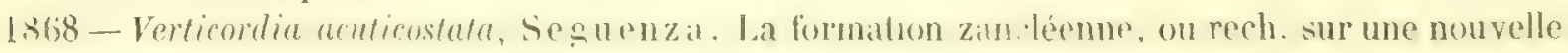
formation tertiaire, p. 475.

1870 - Pechiolia uruticustata. Carpenter e Jeffeys. - Report on Herp-sea researches er.. pag. 158.

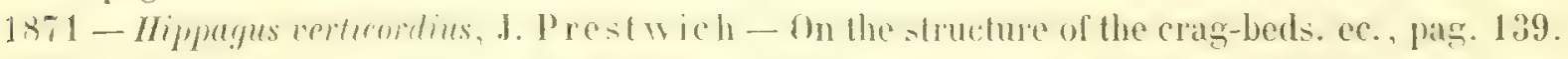

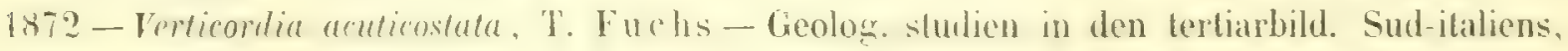
pag. 3 t.

1873 -Verticordia aculicostata, II. C. We inkauff - Catalog der im europaischen ec., p. 56.

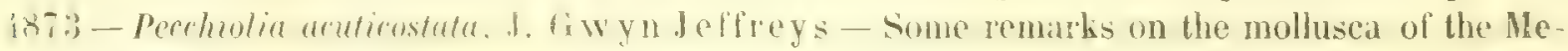
diterr., pag. 116.

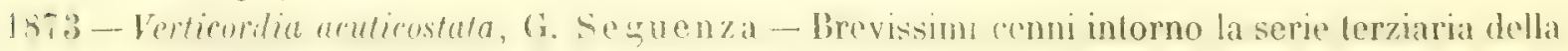
prov. di Messina, pag. 20.

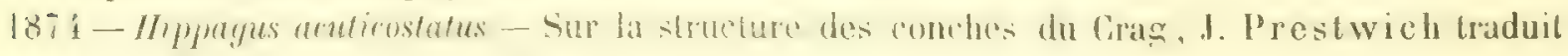
par M. Mourlon, pag. 36.

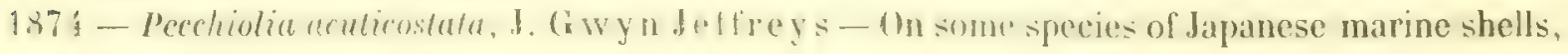
pag. 101.

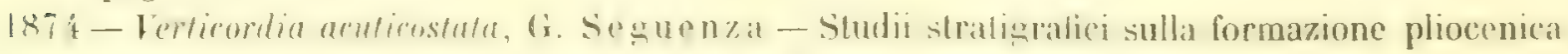
dell' Italia meridionale. (Bollettino del R. Comitato geologico 1874).

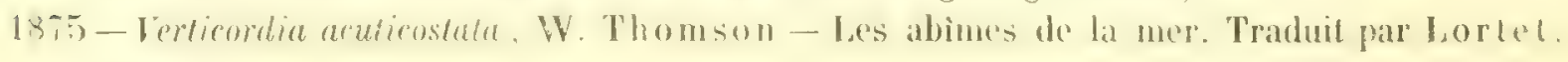
pag. 393.

1875 - Vertirordia acuticostata, Monterosato - Nuova rivista delle conchighlie mediterranee. pag. 15.

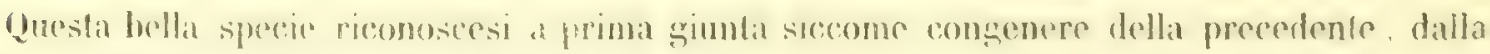

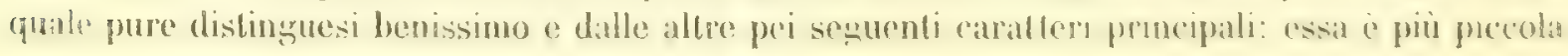


assai della precerdente, costi apter spualmente ripiegalti, ma meno prominenti, colle costole poco

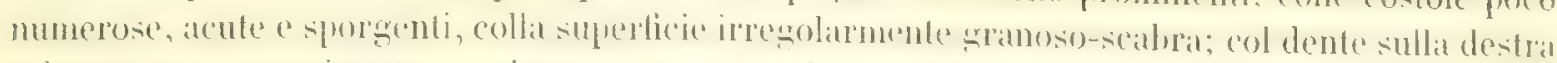

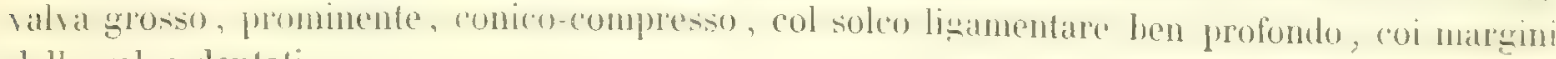
delle valve dentali.

Le più grandi valve misurano una larghezza di $17^{m m}$, ed una lunghezza di $18^{\text {mm }}$

Il Philippi conobbe soltanto la valva sinistra senza denti, e la rapporto al genere Hippa-

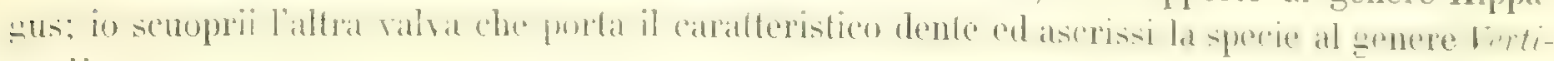
rordia.

Il signor Jeffreys ha identiticato la specie del Philippi colla $V$. Juponica, $\Lambda$. Adams, e colla $V$. Deshayesiana, P. Fischer, ed io non conoscenda queste conchiglie viventi ho seguíto in fede sua tale opinione.

Giacimento. - La $V$. aculicostata fu dapprima scoperta nel Crag d'Inghilterra (S. Wood). Quindi nella valle Lamato in Calabria (Philippi, Costa).

Il suo giacimento ordinario èla zona superiore del plioceno antico (Astiano) deposilato in mare profondo.

Nel territorio di Mlessina si, raccoglie alle contrade Trapani, Scoppo, Scirpi, Gravitelli, S. Filippo, Rometta, Spadafora ec. (Seguenza) (G. S.).

Incontrasi nei dintorni di Raggio (Seguenza) (C.S.); nelle colline di Bianco nuovo (C.S.);

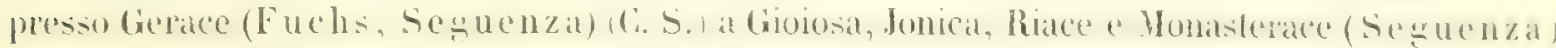
(C.S. S.).

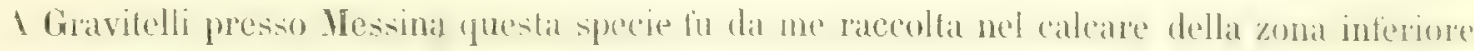
del plioceno recente $(\mathrm{C}$. S).

Molto recentemente ho raccolto ancor'a la $V$. acuticostata nella più recente zona del plioceno

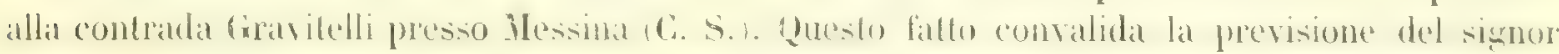
Marc. di honterosato che crede debba questa specie trovarsi vivente nel Mediterraneo.

Vivente è stata pescata nell'Atlantico e nel Nord-Atlantico, siccome nei mari del Giappone.

SECONDO SOTTUGENERE

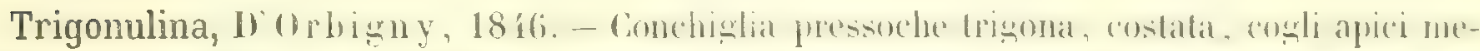
diocremente sporsenti, e fortemente incurvali subla lunula, che e grande e ben distinta; il dente cardinale e ben solido, largo alla base, es'incurval pere innalgarsi vertieatmente sul piamo di riu. nione delle valve; sul marevine posteriore della destra valva e un altro dente molto lareso, poco sporgente, quasi lamelliforme, disposto paralledamente al margine, limitando dal lato interno al soleo del margine dorso-anale. che serve all'inserzione del matremes dell'opposta valva, sul quale è internamente una depressione che riceve l'opposto dente.

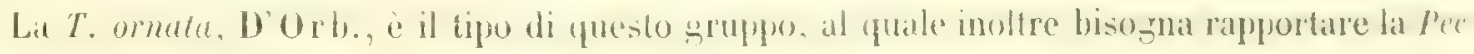
chiolia Gastaldi, Mich., la V. pariensis, Desh.

Terza Specie-VERTicombIA gRINULATA

Sinonimia

1858 - Verticordia granulata, G. Seguenza- Del genere Verticordia. Wood. Breve monografla delle specie fossili di Sicilia (Eco pelorilano. Anno V, fasc. 11 c 12 ) $)$.

1860 -Verticordia granulata, G. Seguenzd - Du genre Verticondia (Journal de Conch. Vol. 8, pag. 299, tav. 10, tig. 2, $f, g, h)$.

1860 - Verticordia gramulata, P. Fischer - Journal de Conch. Vol. 8, pag. 229.

1862 - Verticordia gramulata, P. Fischer-Journal de Conch. Vol. 10, pag, i80. 
1862-Verticordia granulata, G. Seguenza - Notizie succinte intorno la cost. geologica del distretto di Messina; pag. 18.

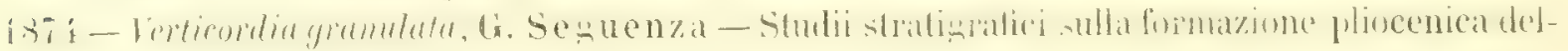

l'Italia meridionale (Bollettino del R. Comitato geologico).

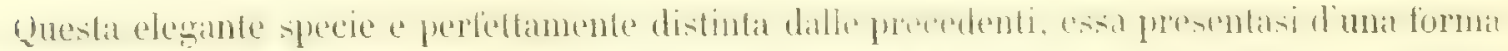

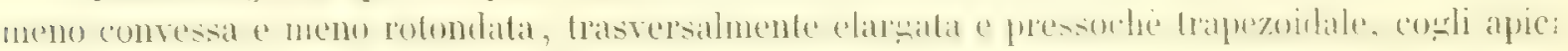
incurvati spiralmente sulla regione boccale, ma assai meno prominenti, colle costole numerose,

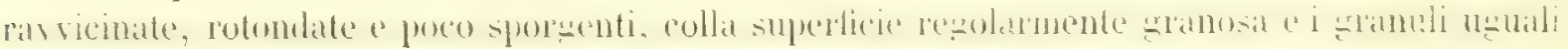
e rotondati. Il dente è ovato-compresso non molto sporgente, i margini di ambe le valve sono leggermente crenate, la lunula è grande, cordata, concava, hen delimitata e senza costole.

La maggiore valva ha un diametro dal margine boccale all'anale di $11^{\text {min }}$, e di $10^{\text {mm }}$ dall'apice il margine ventrale.

Allorquando ho descritto questa specie io possedeva soltanto una valva sinistra, e fu duopo che trascorressero molti anni perchè io potessi raccogliere una valva destra e riconoscere i suoi caratteri.

Giacimento. - La V. yramulata fu da me raccolta nelle marne e sabbie dell'Astiano alle conirade Trapani e Salice nel territorio di Messina (C. S.).

Questa specie è stata indicata siccome vivente in molti luoghi, come al Giappone, in tutto

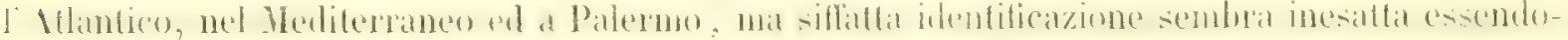

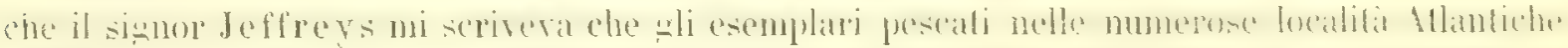

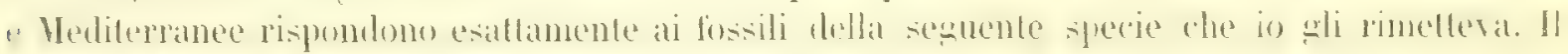

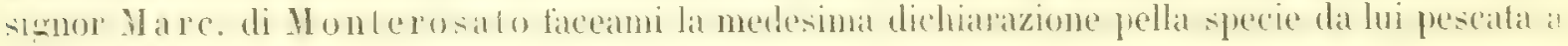
Palermo e pei fossili di Altavilla e di Ficarazi precedentemente riferiti alla V. gramulata.

Wuarta Specie-VERTICOHDL TIAPEZUIUEA.

Sinonimia

1870 -Verticordia gramulata, W. B. Carpenter and J. Givyn Jeffreys - Réport on deep-sea researches ec.

1870 - Verticordia granulala, J. Giwn Jeffreys - Mediteranean mollusca, pag. 9.

1872 - Verticordiagramulata, Mare. di Monterosalo - Notizie intorno alle conchiglie fossiliec.; pag. 24.

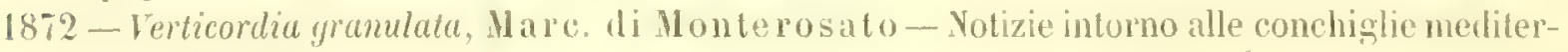
ranee; pag. 22.

1872 - Verticordia granulata, II. C. We inkauff - Catalog. der im europaischen ec.; pag. 56.

1873 - Verticordia gramulatu, J. Gwyn Jeffreys - Some remarlis on the mollusca of the Vediterr.; pag. 116.

187-P - Pecchiolia grumulata, J. Gwy deffreys - ()n some species of Japanese marine shells; pag. 101.

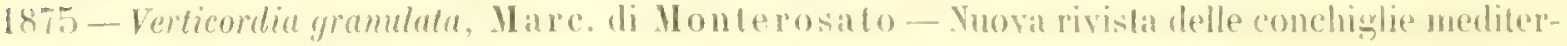
ranee; pag. 15

E questa una specie molto somigliante alla precedente, ma piü piceola piu depressa, di furma esittanente trapezoidale, e cogli apici incurvati verso la regione anteriore, ma piccoli, poco sporgenti non avvolti a spirale. Una oscura piega che parte dallapice percorre la conchiglia diagonalmente sino al margine ventrate dividendoli in due porzioni pressochè uguali, le costole somigliano a quelle della precedente specie, ma sono in minor numero, in quella da "20 a "25, in questa circa 18. Il margine anale si presenti marginato da un rialzo prominente piì delle costole 


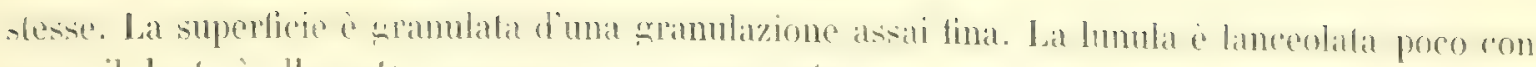
cava, il dente è allargato compresso poco sporgente.

La maggiore valva che possiedo è lunga $4^{\text {man }}$ e quasi ugualmente larga.

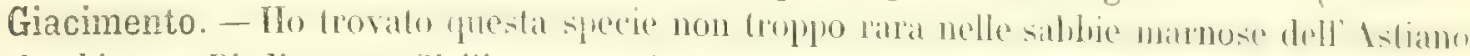
Ira Calatabiano e Piedimonte (Sicilia), e negli strati di Altavilla (C. S.).

Vivente presso Palermo (Monterosato), nel Mediterraneo e nell'Mlanlico (Jeffreys).

Juinta specie-VERTICORDIA ARENOSI

Sinonimia

1868 - Verticordia macrodonta, Seguenza (11. S.) - Nella collezione.

1871 - Pecchiolia arenosa, (Rayneval) F. L. Appelius. Catalogo delle conchiglie fossili del

Livornese desunto ec. ec. (Bull. maluc. ital., Anno III, 1870) pag. 102, Tav. VI, fig. 4.

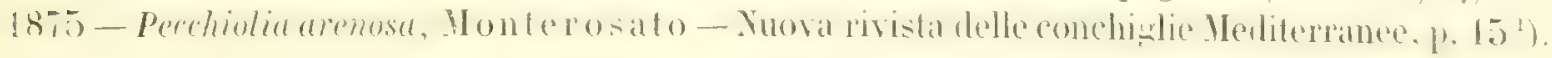

Questa grande specie parmi che debba essere di forma pressochè orbicolata, abbastanza convessa, cogli apici convessi, poco sporgenti, ed incurvati verso la lunula, che è cordato-ovata, ben circoscritta, ablastanza profondir e concats. La superficie e ornata da numerose costole ralianti, strette, disurgali, piu sottili e piu numerose sulla regione anale, alpena sporegenti, mal

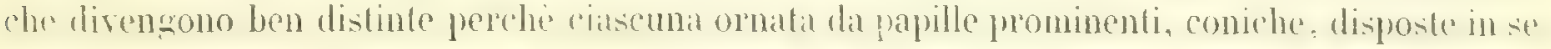

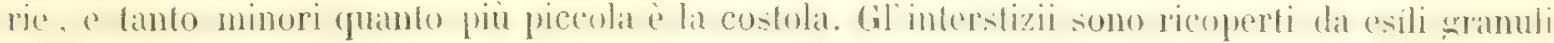
della forma stessa. Il margine interno delle valve è finamente striato o increspato, il dente è

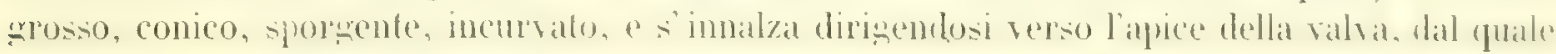
lato presenta uno spigolo acuto.

Questa grande specie raggiunge senza dubbio un diametro di 25 millimetri.

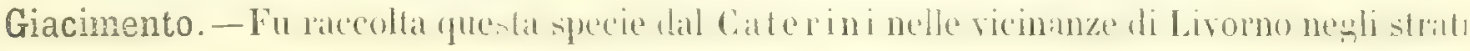
dell' Astiano.

lo posiedeva da lungo tempo un incompleta valva di Gerticordia, raccolla nelle marme delI'Astiano messinese presso Rometta, che teneva nella collezione col nome di $V$. macrodonta, e che ho ravicinato ora alla specie del liayneval (C. S.).

L'anno scorso ho raccolto numerosi frammenti di questa specie nelle marne Astiane delle colline presso Bianconuovo (Calabria) di mita alla $V$. argentea, ed alla $V$. acuticostata, e nelle stesse condizioni poco fa la trovava a Gioiosa jonica, kiace, Monosterace (C. S.).

Se la Pechiolia exasperata, Ponzi, non ancora descritla, risponde a questa specie, può dirsi che essa giace anco nelle marne del Vaticano.

Il Marc. Monterosato pescò nel mare presso Palermo alcuni frammenti d'una Verticordia che dubbiosamente riferisce alla $V$. arenosa.

\section{TERZO SOTTOGENERE}

Laevicordia, Seguenza, 1876. - Conchiglia senza costole, di forma che si arvicina alla

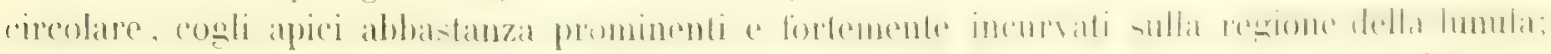
il dente cardinale è più o meno largo, compresso e sembra più tosto una prominenza del mar-

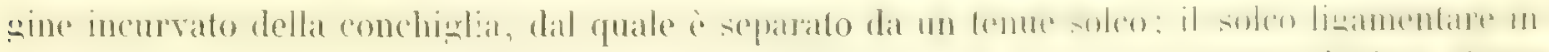

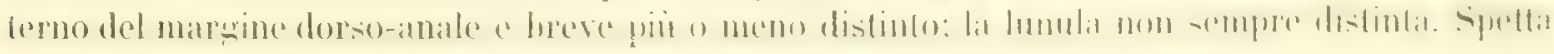
probahilmente a questo gruppo la $V$. abyssicola, Sar's. 
Sesta Specie - VERTICORDIA INSLULPTA

Sinonimia

1862 - Verticordia ecostatu, Seguenza (II. S.) - Nome imposto nella collezione.

1873 - Verticordia insculpta, Jefreys - Réport Brit. Assoc., p. 112.

1875 - Verticordia insculpta, Monterosato - Nuova rivista delle Conch. mediterranee, pag. 15.

Questa piccola specie ha le sue valve sottili e molto fragili, di forma orbicolato-trigona.

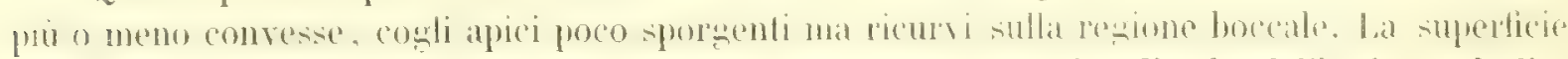
delle valve è segnata da soltili linee impresse che la percorrono irradiando dall' apice e da lievisime punteggrature o asprezze che si osservano soltanto con una lente di forte ingrandiunente.

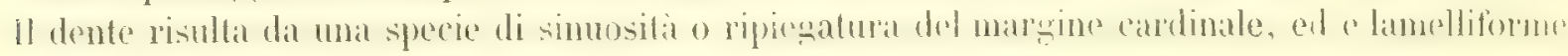
e poco prominente. La lunula non è distinta.

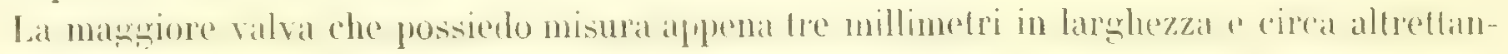
to in lunghezza.

Da ben tredici anni possiedo questa specie nella mia collezione col nome di $V$. ecostata, $\mathrm{e}^{\mathrm{a}}$

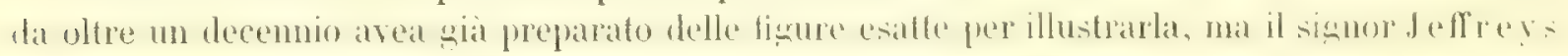
la pescava recentemente nel Jediterraneo denominandola $\mathbf{V}$. insculpta.

Giacimento. - Mllo stato fossile questa fragile specie mi ha offerto qualche valva più o meno incompleta nelle marne astiane delle contrate frapani e Rometta nel Mesinese (C. s...

II Jeffreys pescava questa Verticordia sulle coste d' Algeria, e presso Palernı il M arc. di Nonterosato.

Settima Specie-VERTICORDLA MYTLLOLE

Sinonimia

$1876^{\circ}$ - Verticordia mytiloides, Seg. - (M. S.) Nella collezione.

Conchiglia cordato-trigona formita di pieghe concentriche. pel quate calatlere e per la forma

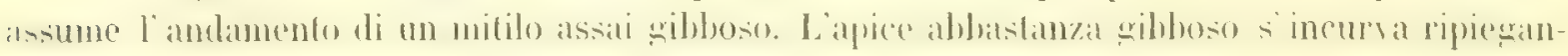

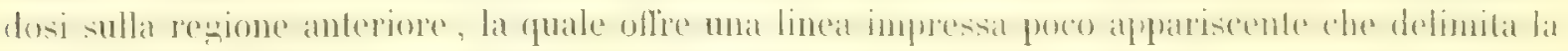

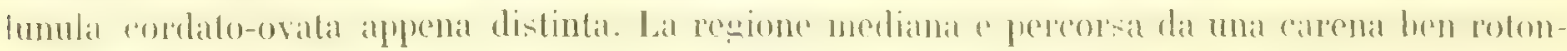

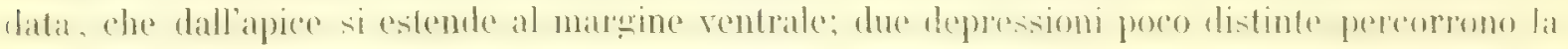
valva dall'uno e l'altro lato della carena rendendo questa meglio prominente e più distinta; ed alla loro volta entrambe vengono seguíte all'esterno da lievi rialzi, di cui l'uno scorre sul marsine dorso-anale e l'altro molto arrotondato cinge la regione della lunula. La superficie esterna non ben conservata sembra dovere essere granulata finamente.

Giacimento. - La ralva sinistra che possiedo ì stata raccolta recentemente in un calcare ustiano di Spadafora ('Terr." di Messina).

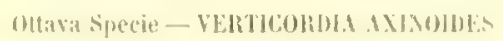

Sinonimia

1876 - Verticordia axinoides, Seguenza - (I. S.) Nella collezione.

Grande conchiglia giblosia cordato-trigona, axiniforme per una simuosita assal forte che forma un angolo rientrante marcatissimo, il quale percorre le valve dall' apice al margine ven- 
trale sulla regione posteriore, separando dal resto la porzione anale. La regione boccale è anch' essa cinta da una lieve sinuosità prominente, la parte media delle valve è percorsa longitu-

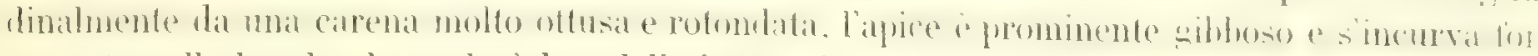
temente sulla lunula, la quale è ben delimitata, piccola, cordiforme, concava e fortemente in-

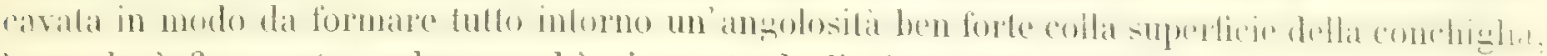
la quale è finamente scabra perchè ricoperta da lievi rugosità trasversali miste arl una tenue granulazione. Il dente risula da una vera ripiegatura del margine cardinale, ed è perciò allargato, lamelliforme e poco prominente; il solco ligamentare abbastanza largo e profondo.

Questa specie, la piu grande del genere, raggiunge approssimativamente la lunghezza di $30^{m+n}$, e lo spessore di circa $35^{m+n}$.

E distintissima per la sua forma speciale, rassomigliante ad un grande axims molto conresso.

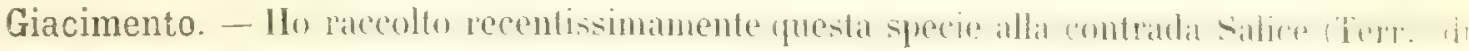
Ilessina) nelle sabbie dell'Astiano, e non possiedo sịn'ora che una valva destra mutilata, e laluni altri frammenti (C.S.).

Vuna suecie-VERTICORdi ORBICULATA

Sinonimia

1862 - Verticordia orbiculata, Seguenza - (II. S.) Nella collezione.

Questa specie è depressa di forma quadrangolare e quasi circolare, cogli apici piccoli mał

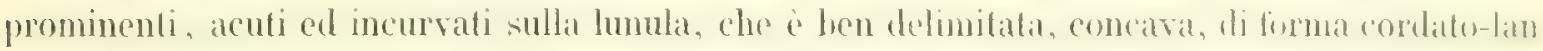

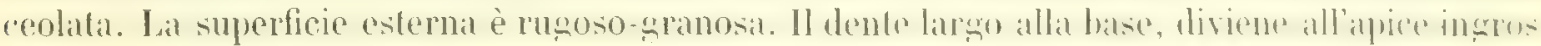

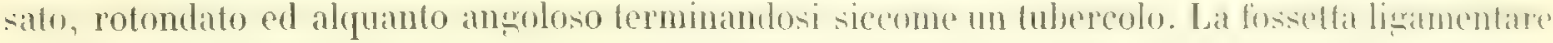

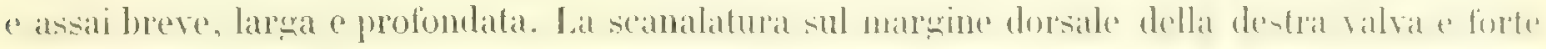
mente distinta, ed e limitata internamente da una prominenza lumzal, lamelliferme, rialzatas: tales scanalatura risponde ad una depressione esterna sul margine dorsale dell'oppostil valva.

Le maggiori valve hanno lunghezza e larghezza di circa otto millimetri.

Giacimento. - Questa specie, dhe possiedo sin da lungo lempo mella mia collerione" a stata

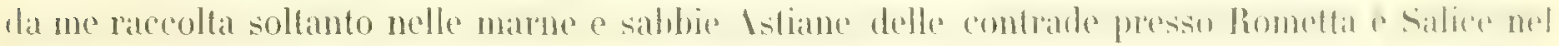
T'err. ${ }^{0}$ di Messina (C. S.).

\title{
i) i certe poce vuleaniche interstratificate fra rocee di sedimento
}

\author{
in $\mathbf{T}$ \\ del Socio Corrispondente nazionale Giuseppe Seguenza
}

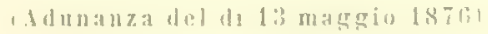

Sin da qualche tempo mi vennero oflerti da varii amici laluni esemplari di una roccia, al fine di sludiarla, che a prima giunta riconoscesi agevolmente per un deposito di ponici leggermente cementato. Tali campioni si aveano varie provenienze, ma sempre da contrade che stanno

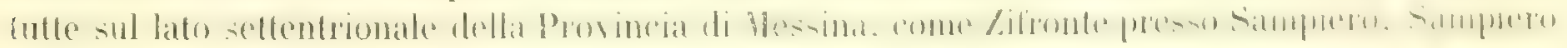
di Patti ecc. Il Signor Ingegnere Lodovico Molino F' oti aveami annmciato ancolat che nelle argille plioceniche presso Barcellona, le (juali si estendono a costiluir la pianura, raceolqonsi delle pomici talvolta abbondanti. 
Questi diversi fatti, quantunque fossero stati da me riguardati siccome importantissimi sin lal primo momento che ebbi ad apprenderli, pure non erano slati da me constatali ancora per-

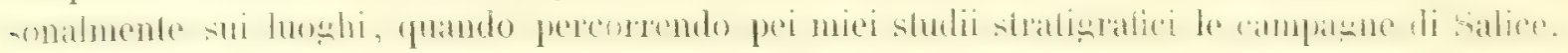
villaggio posto sul versante settentrionale della piecola catena peloritana, nello scorso anno m' imbatteva in una serie di strati marnosi e sabbiosi spettanti alla zona superiore del plioceno antico, depositati in mare profondo, i più ricchi di fossili che io conosca in ambe le provincie

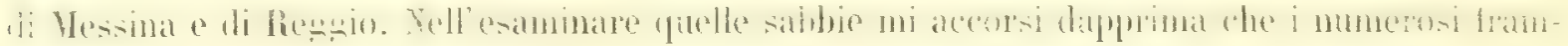
menti neri, misti ai grani quarzosi e calcarei e ad un maggion numero di fossili variatissini e dei loro frantumi, spettano senza dubbio a roce rulcaniche.

Quindi trovai sparsi in certi strati e con abbondanza frammenti di pomici, e finalmente tro-

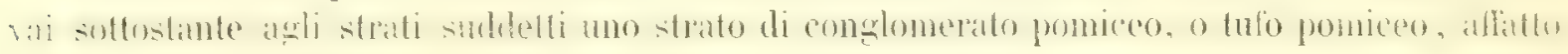
identico a quello di Zifronte, Sampiero di Patti ed altri luoghi.

I pochi fatti esposti sono sufficienti a chicchessia per indagare di quei materiali vulcanici

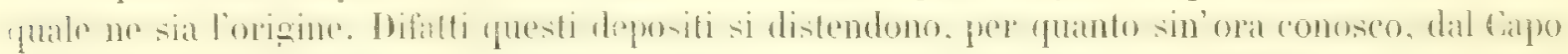
Tindaro al Faro di Vessina: essi mancano sul versante opposto dei monti Peloritani, essi sono

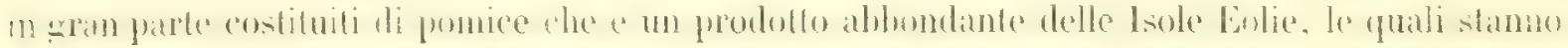

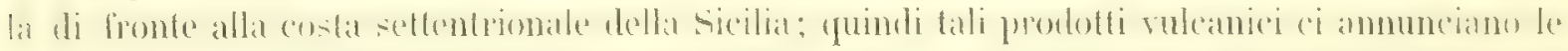

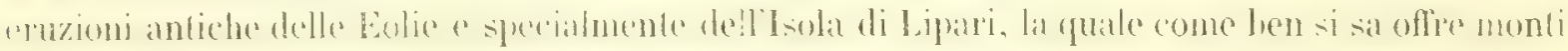
(s correnti, e conglonerati di pomice, quindi uno studio accurato stratigrafico ci scuoprirà l'età

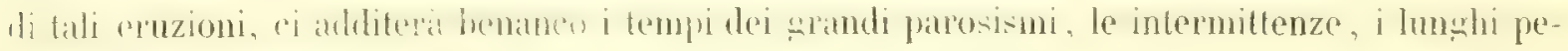
liodi di riposo ecc. ecc.

Un tale studio non mi è stato possibile di compierlo, esso non è che iniziato, ma pure ba-

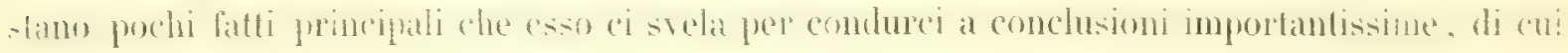
non saprei protrarre oltre l'annuncio.

Io non conosco sin'ora un lnogo dove l'associazione di rocce eruttive e di strati sedimentarii con fossili d'ogni classe possa meglio seguirsi che al Salice.

La è una serie di strati non interrotta e regolarissima di età ben note, dal mioceno medio agli

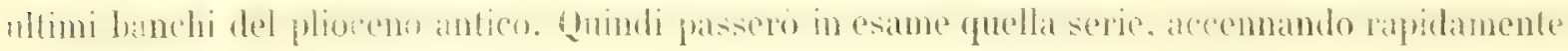
i fatti che ci offre. Una sezione naturale alla scala di $\frac{1}{10000}$ farà bene apprendere la successione stratigrafica e la posizione relativa dei varii prodotti vulcanici.

La serie degli strati poggia sulla formazione cristallina ed è costituita dal basso all'alto nel modo che siegue:

$1^{0} q$ Conglomeralo di ciolloli e ghiaie di rocee cristalline, senza fossili, che spella all' età del mioceno medio.

$2^{\circ} p$ Argille e molasse con una grande varieti dell'ostrea cochlear, Poili, del mioceno superiore.

$3^{\circ}$ o Calcare concrezionato di colore rostastro ovvero bianco, molto potente, che forma il primo membro del plioceno.

$4^{0} n$ Marne bianchicce a forminiferi, le quali della potenza di circa trenta metri racchiudono Ellipsoidina ellipsoides, Nodosarie, Cristellarie, Rotaline, Orbuline, Globigerine ecc., che costituiscono gran parte della roccia, nella quale ho raccolto anco i frammenti della lihynchonella bipartita, Brocech i.

Quest'ultimi due menbri che sono i primi del plioceno costituiscono la zona inferiore del

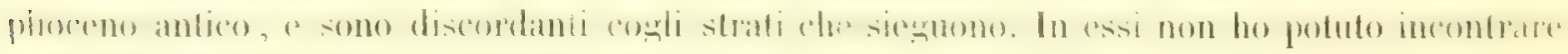
sin'ora detriti vulcanici di veruna natura.

$5^{0} \mathrm{~m}$ Strato calcareo di variabile spessore da mezzo metro a due, con fossili poco riconoscibili (Coralli, Brachiopidi).

$6^{0}$ l Una roccia mamosa gralliecia, ricca di foraminiferi, spessia appena un metro, che lacehiude Terebratula Meneghimian, Se ve., Terebratulina Guiscurdiana, Seg. . specie ben caratteristiche della zona superiore del plioceno antico. 
io Una roccia polverulenta, o almeno molto friabile, bianca, semischistosa, molto ruvida al tutto, che forma una zona di oltre un metro senza fossili.

Questa roccia nella nedesima posizione stratigrafica mi era gỉ nola sin da lungo tempo. avendola incontrata a Rometla ed a Collaina presso Capo Tindaro. La sua natura mi era sconosciuta alfatto; essa contiene pochissimo calcare, e la parte principale è formata da polvere che gli acidi non attaccano, ma che dopo la digestione in essi, trattata sola al camnello offre chiaro

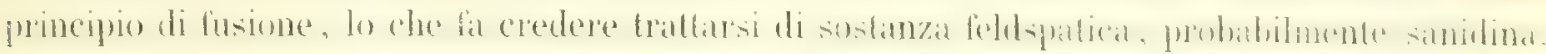
dipendente dal disfucimento di rocce trachitiche. In ogni caso è evidentemente un detrito di origine vulcinica.

$8^{0} i$ Uno strato meno spesso di un metro di marna nero-bruna, contenente considerevole quantiti di acerlesio, che è quello che la colora, ed insieme quella medesima sostanza abbondante che forma lo strato precedente; non ho incontrato alcun fossile in questo strato.

$9^{9}$ h Marne sabbiose giallastre che sembrano proprio quelle dello strato l, e che raggiungono lo spessore di tre a quattro metri: contengono Limopsis mimula, Phil., Nucula sulcata.

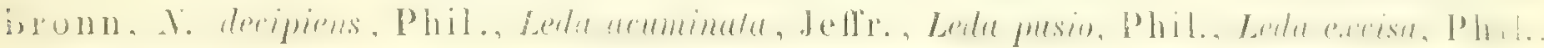

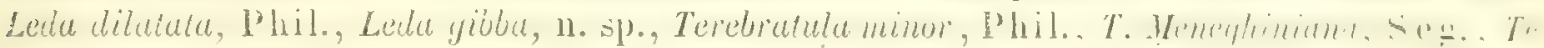
rebratulina Guiscardiana, Seg., Terebratella septata, Phil., ece.

$10^{\circ}$ y Strati marno-sabbiosi grigiastri della polenza di circa quindici melri, che racchiudono gran quantita di foraminiferi ed alcune delle Lede degli strati precedenti.

Gli strati $h$ e $g$ della potenza complessiva d'una ventina di metri non cí offrono apprezza bili detriti di rocce vulcaniche.

$11^{\circ}$ f Succede quindi uno strato sabbioso ricchissimo delle valve del pachylasma gigan teum, Plit.

$12^{\circ}$ e Quindi una serie di strati sabbiosi sciolti, alternanti con altri cementati dal calcare.

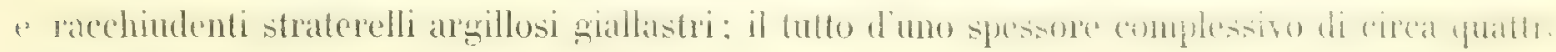
il cinque metri.

In questi strati trovansi in abbondanza frammenti di pomice e di dolerite or compatta ed or più o meno cellulosa. E da notarsi che i frammenti sono angolosi e non mai arrolondati dal-

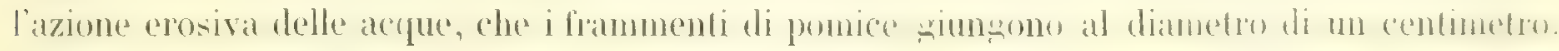
e quelli di dolerite sono di tutte le dimensioni sino a quattro o cinque millimetri. l'ra i lossili che vi sono misti ricorderò: Verruca zanclea, Seg., V. dilatata, Seg., V. crebricosta, Seg., Rimula radiata, Lib., Limopsis minuta, Phil., Arca aspera, Phil., A. tetragona, Poli, Leda ¿byssicola, Torell, Terebratutina caputserpentis, Lin., Waldheimia cranimm, Mül., Conocrimus Seguenzai, Me negh., ecc.

$13^{\circ}$ a Tufo pomiceo di un metro e mezzo di spessore, il quale contiene frantuni des soliti fossili, ma non vi si osservano frammenti di dolerile o di pirosseno. I pezzi di pomice che costituirono questa roccia sovente sono ben discernibili, in altri casi è una massa assai fraçile, friabile, quasi uniforme.

$14^{\circ}$ c Sabbie più o meno agghlutinate e grossolane, ricchissime di fossili d'ogni clatsoc.

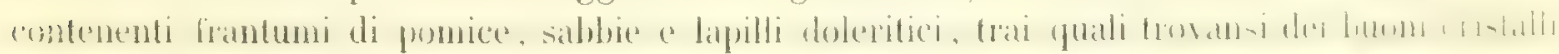

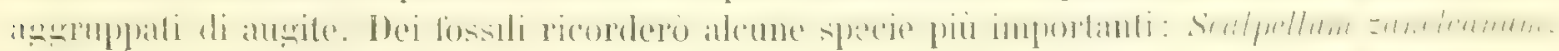
Seg., S. Michelottianum, Seg., Scillaclepas carinata, Phil., S. ormuta, Seg., Vormet stromia, Vüller, V. zanclea, Seg., V. dilatata, Seg., V. Romeltensis, Seg., V. crebricostu, Seg., Pachylasma giganteum, Phil., Columbellu costulata, Cantraine, Triforis perverse, Isanki., Trochus bullatus, Phil., T. semigramularis, Cantraine, T. Ottoi, Phil., T. Margimulatus, Phil., T gemmulatus, Phil., T. clathratus, Aradas, T. Laciue, n. sp., (Otivia Tinei, Calcara. Turbu pelorilaus, Cantraine e varieli, T. Romellensis, Seg., Solarium hemisphaericum, Sex.. Jlelu tenella, Jefrr., kissoa cimicoudes, Forbes, Eulimella Scillue, Scacchi, Fissurella lomuirla thrata, Seg., Fissurisepta papillosa, Seg., F. rostrata, Seg., Puncturella nouchina, hi in.., ki-

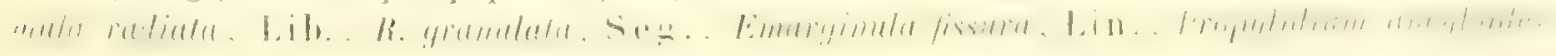


Forbes, Dentalium agile, Sars., Siphonodentalium tetragonum, Brocchi, Gadus subfusifol-

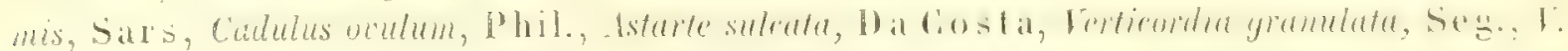
aculecostata, Phil., Area pectunculoides, Scacchi, A.aspera, Phil., A.obliqua, Phil., A.tetrayona, Poli, Limopsis aurita, Brocchi, Nucula sulcala, Bronn, Modiola phaseolina, Phil., Limea Sarsii, I. ov., Pecten vitreus, Gm., P. islandicus, Lin., Anomia aculeata, Phil., Terebratulu vitrea, Born., T. minor, Phil., Terebratulina caputserpentis, Lin., Waldheimia cramim, Mülle r, W. septigera, Lowen, Megerlia truncala, Gm., Platydia anomioides, Sc., Argiope decol-

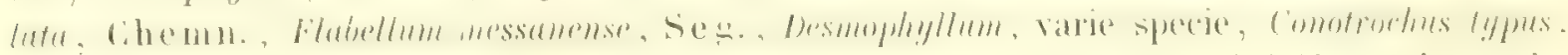
Seg., Ceratocyathus communis, Seg., Steplanocyathus elegans, Seg., Lophoheliu, varie specie. Diplohelia, ecc. ecc.

Lo spessore di questa zona è di circa dieci metri.

$15^{\circ} \mathrm{b}$ Banchi di coralli che si sono costituiti in roceia calcarea compalta. Il corallo princi-

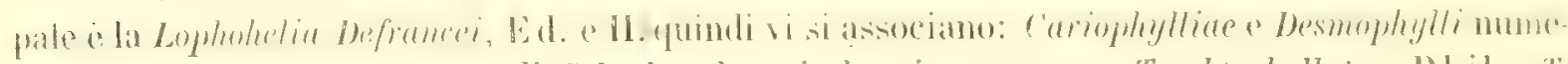
rosi, e conchiglie di Gasteropodi Solarium haemisphaericum, n. sp., Trochus bullatus, I' hil., T. semigramularis, Gantr., Trochus Maurolici, n. sp., ecc. ecc.

La potenza di tali banchi di coralli giunge a circa sei metri.

Dallo strato $m$ allo strato $b$ abbiamo una serie variatissima di strati, che costituiscono unico membro del Plioceno, cioè il superiore del plioceno antico.

Finalmente un lembo di alluvione antica a ricuopre l'ultimo strato dell'antico plioceno, senza

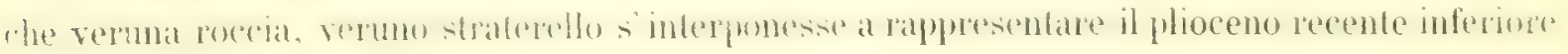
o superiore clie sia.

Dai fatti precedentemente esposti ne derivano chiaramente le seguenti conchiusioni:

$1^{0}$ La serie stratigrafica di Salice dimostra evidentemente delle eruzioni avvenute nelle

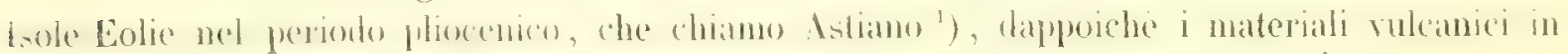
essa osservati trovansi negli struti della zona superiore del plioceno antico (Astiano).

$2^{\circ}$ Ad un passegrgiero esame delle rocce mioceniche e del pii antico plioceno (Zancleano)

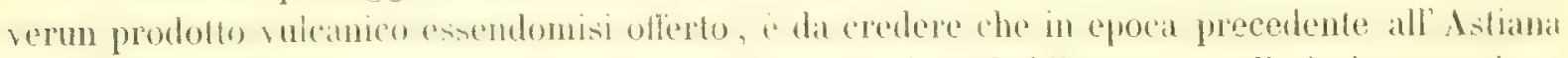

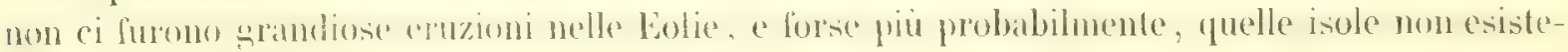
vano ancora.

$3^{0}$ In sul cominciare del periodo Astiano noi troviamo gli strati $k$ ed $i$ formati principalmente da sostanza vulanica polverulenta che annuncia evidentenente una enorme eruzione, ac-

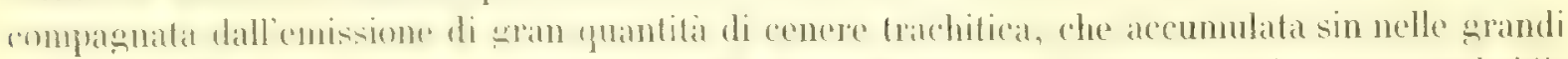
fonfondita del mare enstituina teposili di piu metri di spessore. Siffilta eruzione cra probabil-

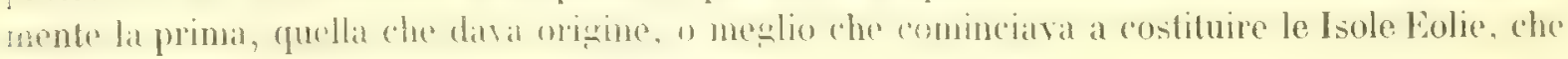
vedonsi in parte formate da rocce trachitiche.

$1^{0} 1$ questo periodo di esplosione ne sucereleva uno di rijoso, esisendoche vediamo nebla

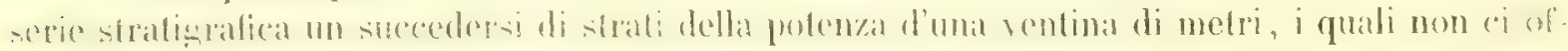
frono prodotti vulcanici, ed invece alla base racchiudono le specie stesse di fossili che ci sono negli strati che precedono il detrito trachitico.

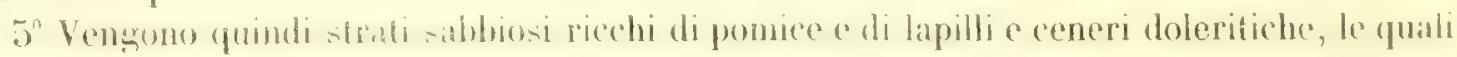

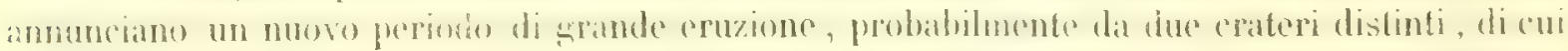

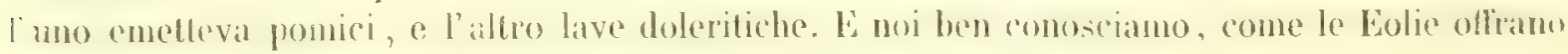
crateri, correnti ed accumuli doleritici e pomicei.

$6^{\circ}$ La cruzione pomicea pare che poscia abbia preso maggiori proporzioni, da costituire un deposito di sole pomici di oltre un metro e mezzo.

$7^{0}$ Quindi decrebbe mentre si continuava la eruzione doleritica. 


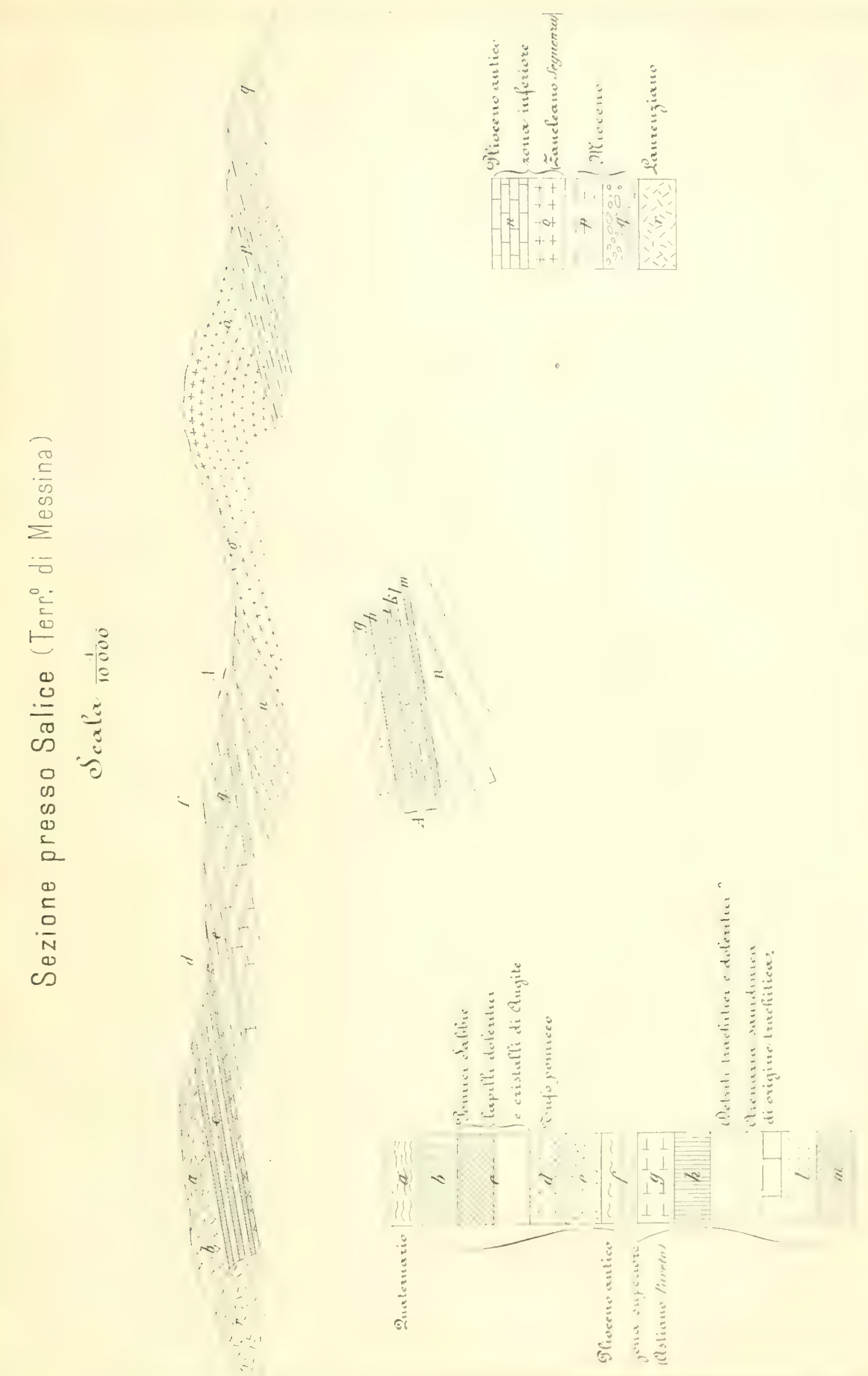


.

. 

. 

2.3 


\section{reace

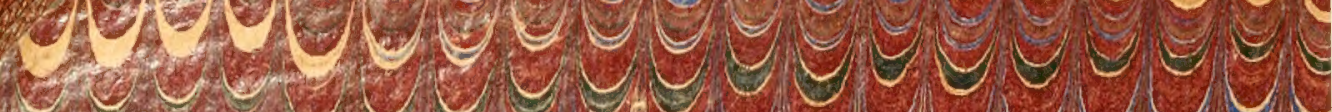

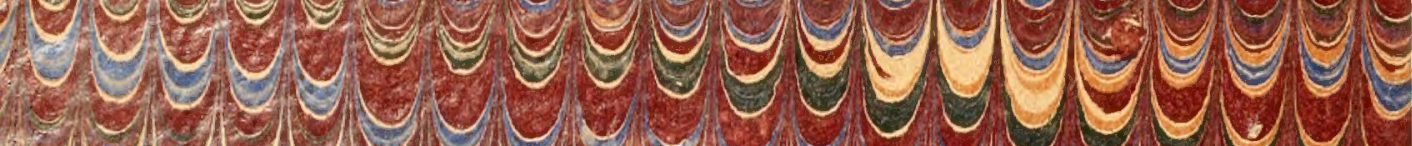
(5) (3) (

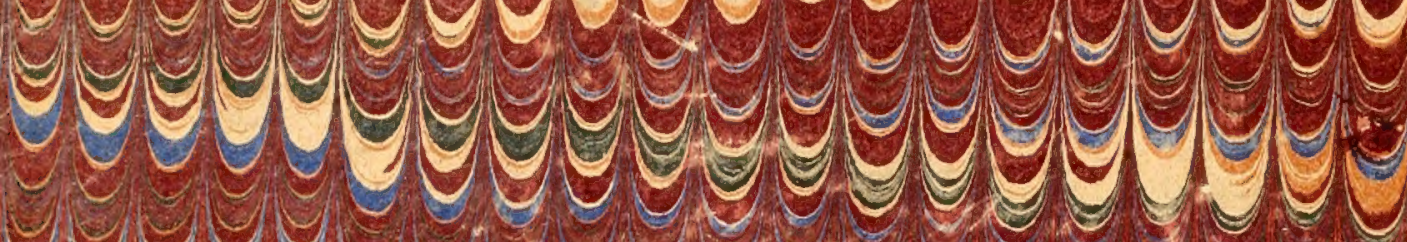

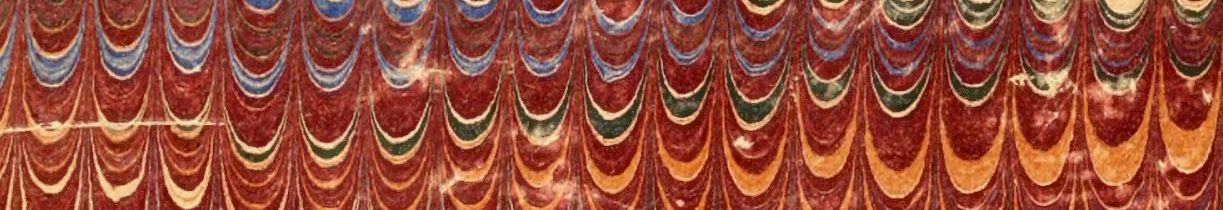

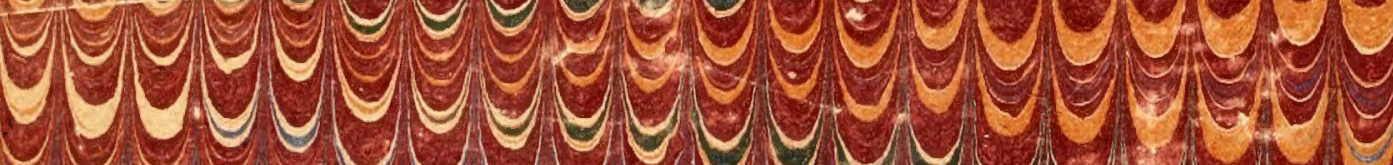

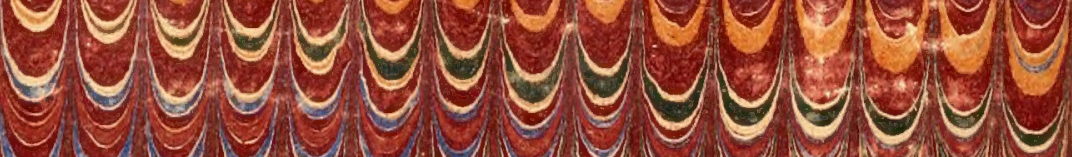

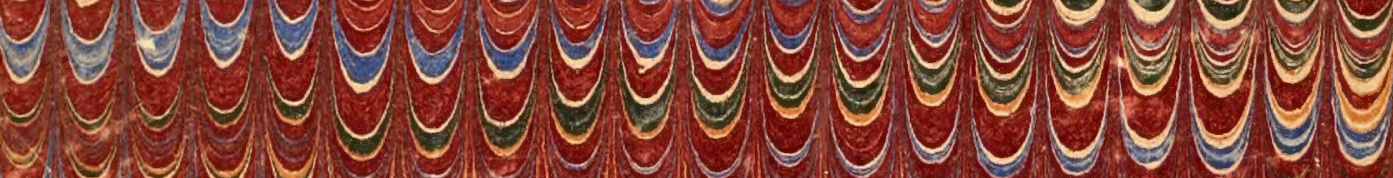

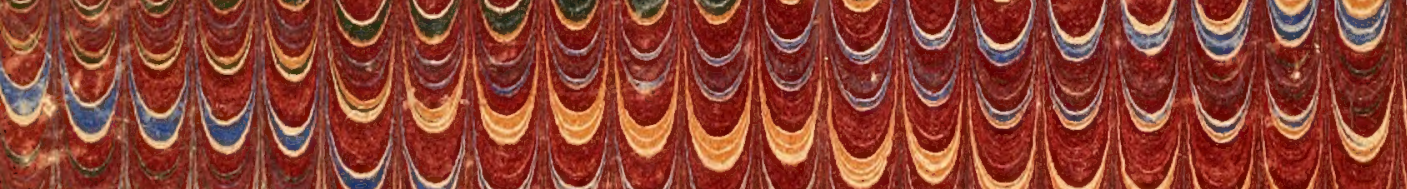
-

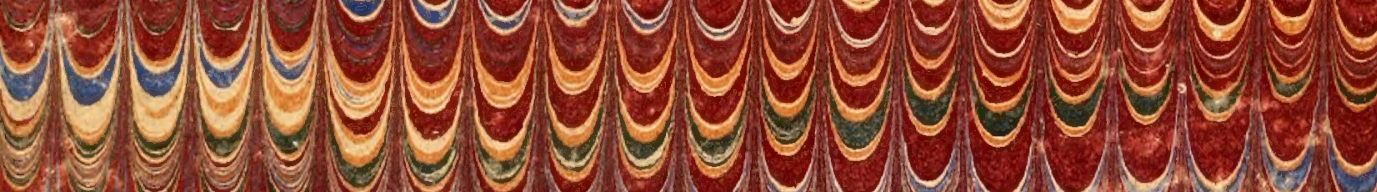
S

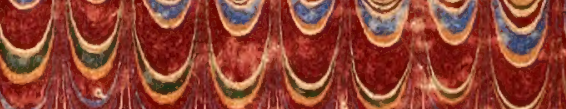

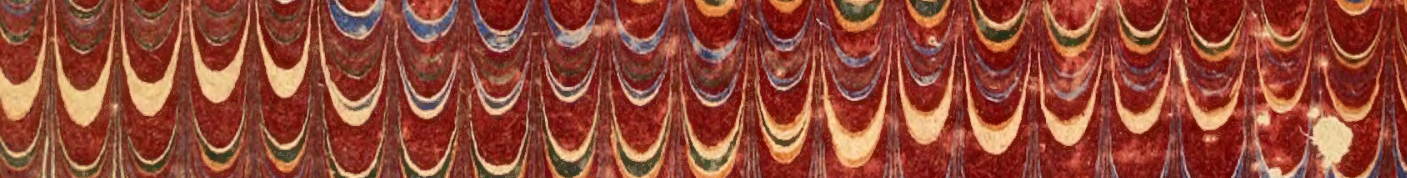
C y y y a _

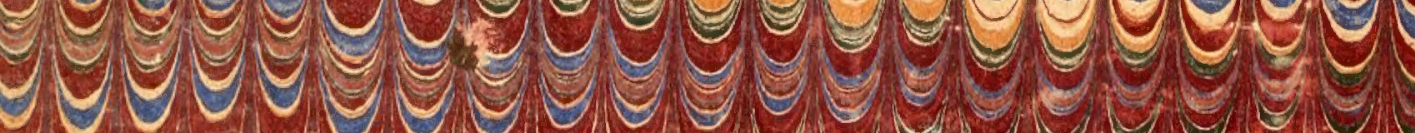

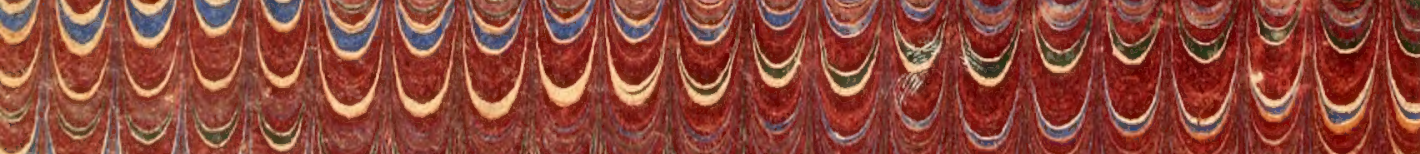

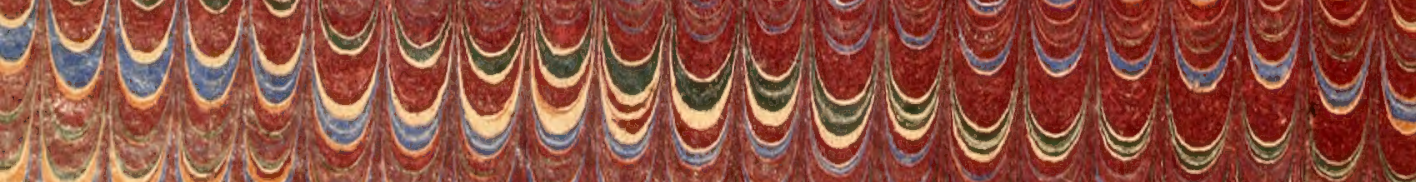
- g y Y.458

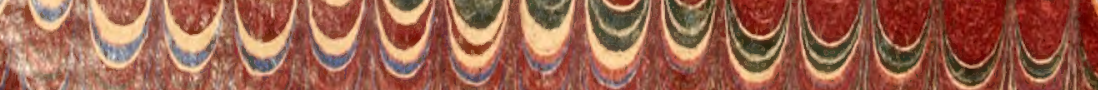

